\title{
Influence of UTA-Si buffer layers on the growth of SiGe films analyzed by high resolution X-ray reciprocal space map*
}

\author{
S.Q. Zheng, ${ }^{\dagger}$ M.M. Rahman, M. Kawashima, M. Mori, T.Tambo, and C. Tatsuyama \\ Department of Electrical and Electronic Engineering, Faculty of Engineering, \\ Toyama University, 3190 Gofuku, Toyama 930-8555, Japan \\ (Received 12 November 2004; Accepted 17 December 2004; Published 24 December 2004)
}

\begin{abstract}
The $\mathrm{Si}_{1-x} \mathrm{Ge}_{x}(x \approx 0.25)$ films were grown on $\mathrm{Si}(001)$ substrate using ultra-thin amorphous $\mathrm{Si}(\mathrm{UTA}-\mathrm{Si})$ buffer layers with thickness from $0 \sim 30$ Åby molecular beam epitaxy. The films were mainly characterized by a reciprocal space mapping performed on a high-resolution X-ray diffraction. It is shown that the smooth and relaxed SiGe alloy layers are obtained using UTA-Si buffer layers, without the necessity of using several microns of thick compositionally graded layers. The surface morphology and crystal quality of SiGe thin film can be improved obviously. The strains in both [001] and [110] directions were relaxed greatly depending on the thickness of UTA-Si.

[DOI: $10.1380 /$ ejssnt.2004.256]
\end{abstract}

Keywords: Molecular beam epitaxy; high-resolution x-ray reciprocal space map; SiGe film; UTA-Si

\section{INTRODUCTION}

SiGe is now a material being broadly employed in CMOS, bipolar, optoelectronics, sensors, and a variety of other areas due to its useful optical and electronic properties. To realize the utilization of $\mathrm{SiGe}$, strain relaxed and smooth alloy layers are required. However, The large lattice mismatch of about $4.3 \%$ between Ge and $\mathrm{Si}$ has limited the growth of high-quality SiGe alloys to within a certain thickness, the so-called critical thickness, and often results in high threading dislocation (TD) density even up to $10^{11} \mathrm{~cm}^{-2}$ in the SiGe layers grown on $\mathrm{Si}$ substrates without buffer layers. These TDs can propagate through the SiGe layers into the electronic active layers on top, degrading the electrical and optical performance of the devices. To date, several types of buffer layers have been used to grow relaxed SiGe layers having a low TD density, including compositionally graded $\mathrm{Si}_{1-y} \mathrm{Ge}_{y}(0<y<x)$ layers [1], $\mathrm{Si} / \mathrm{Si}_{1-y} \mathrm{Ge}_{y}(y<x)$ superlattice [2], graded short-period $\left(\mathrm{Si}_{m} \mathrm{Ge}_{n}\right)_{N}$ superlattice [3-5] and low-temperature grown $\mathrm{Si}(\mathrm{LT}-\mathrm{Si})$ layers [6-9], and so on. However, the buffer layers have to be relatively thick in order to achieve low dislocation densities and strain relaxation for device applications. Among the techniques for the growth of SiGe, the low-temperature grown $\mathrm{Si}$ buffer layers are widely used to reduce TDs and improve surface morphology in the SiGe alloy layers, which might be due to an enhanced defect incorporation/ injection effect. Lee, et al [8,9] studied the effects of the thickness and growth temperature of LT-Si buffer layer on SiGe growth and pointed out that $200 \mathrm{~nm}$-thick buffer layer grown at $400^{\circ} \mathrm{C}$ is better for SiGe growth. In our previous research, we have shown that short-period $\left(\mathrm{Si}_{m} / \mathrm{Ge}_{n}\right)_{N}$ superlattices with various steps are effective to reduce the surface roughness and strain [3]. However, it needs to increase the buffer thickness in order to obtain strain-free and smooth SiGe alloy layers. But, for device fabrication, such as the monolithic integration of

* This paper was presented at The Sixth Japan-Russia Seminar on Semiconductor Surfaces (JRSSS-6), Toyama, Japan, 10-17 October, 2004 .

†Corresponding author: zhengsq@eyou.com devices, the thinner buffer layer will be better. So, we are paying our attention to get effective and thinner buffer layers, which will lead lower cost and minimum time for fabrication. In this paper, we succeeded in fabricating SiGe alloy layer with ultra thin amorphous Si (UTA-Si) as buffer layer and studied its effects on SiGe top layer mainly by high-resolution X-ray reciprocal space mapping (HRRSM) measurement.

\section{EXPERIMENTAL}

All samples were grown on $\mathrm{Si}(001)$ substrate in a molecular beam epitaxial (MBE) chamber (ANELVA, E-620s) equipped with reflection-high energy electron diffraction (RHEED) and X-ray photoelectron spectroscopy (XPS). Un-doped high purity (10-nine) Si and Ge were evaporated from an electron beam evaporator and a Knudsen cell (K-cell), respectively. The substrate was cut into $25 \times 25 \mathrm{~mm}^{2}$ from $0.625 \mathrm{~mm}$-thick $\mathrm{Si}(001)$ wafers. It was heated by a carbon heater, and the substrate temperature was measured by a W-Re thermocouple attached

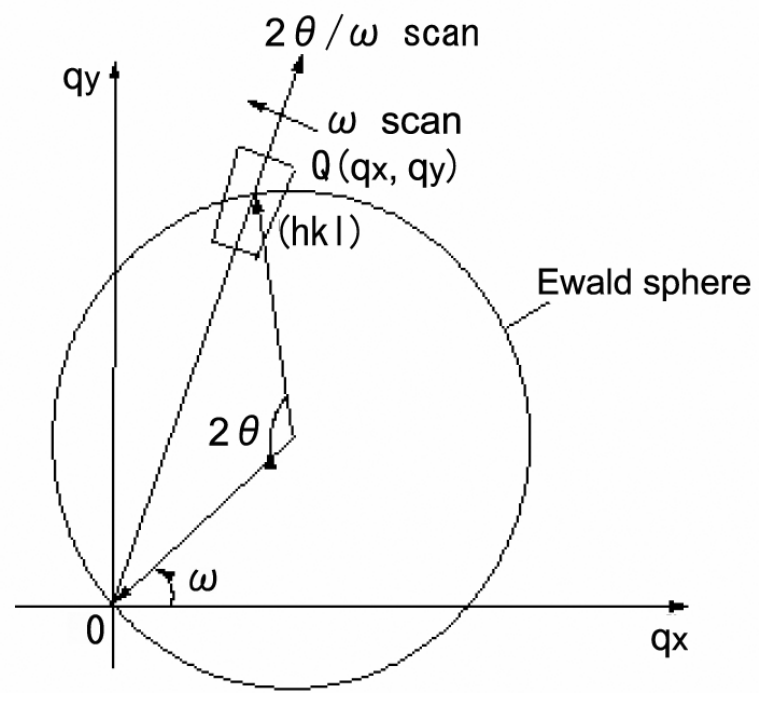

FIG. 1: The reciprocal space coordinates. 
(004)RSM

(a)

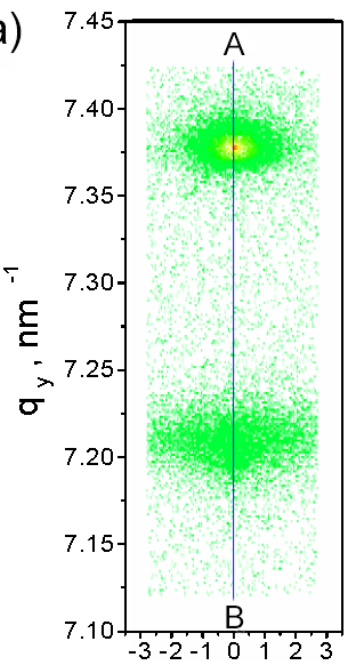

(b)

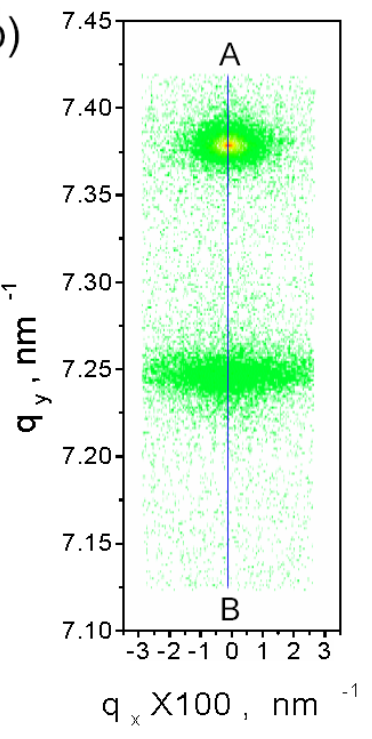

(113)RSM
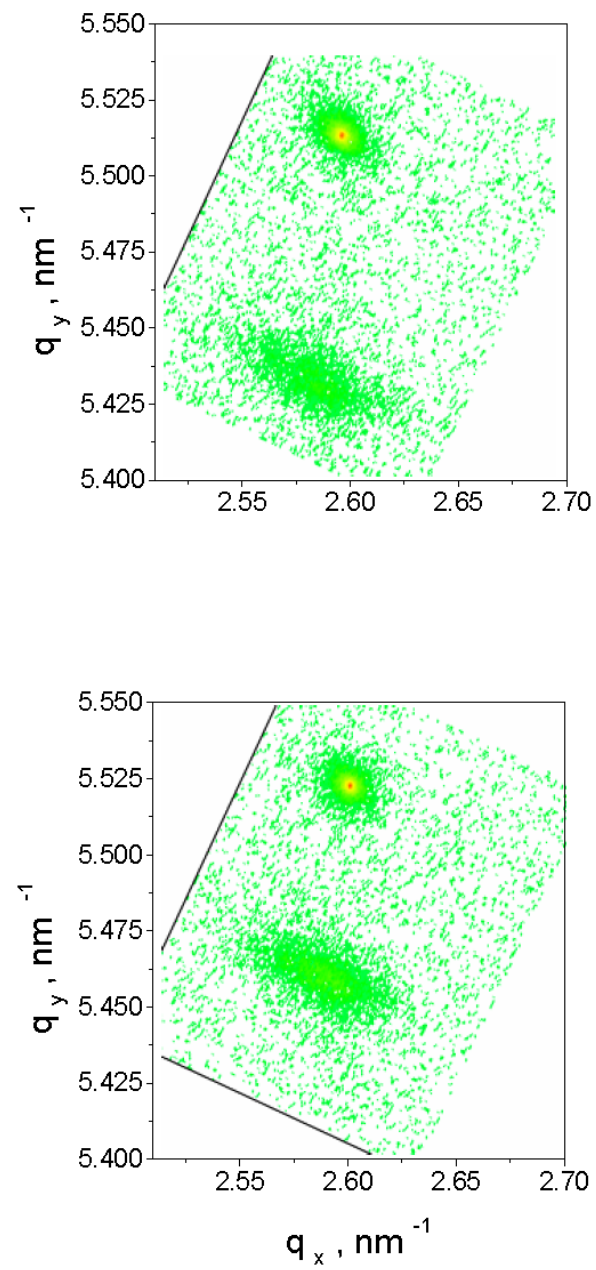

FIG. 2: The typical reciprocal space maps of the (004) and (113) reflections from (a) SiGe/Si, (b) SiGe/UTA-Si(20 A)/Si.

on the substrate holder. The growth rate estimated by a quartz thickness monitor (Model: INFICON IC 6000) was about $0.5 \AA /$ s for Si. After the standard chemical cleaning process, the substrate was moved into the MBE chamber, where the substrate was annealed at $850^{\circ} \mathrm{C}$ for $20 \mathrm{~min}$ to remove $\mathrm{SiO} 2$. After depositing 1000 - $\AA$-thick Si at $750^{\circ} \mathrm{C}$, the substrate was again annealed at $850^{\circ} \mathrm{C}$ for $20 \mathrm{~min}$. After these treatments, the surface exhibited a clean $(2 \times 1)$ RHEED pattern. The samples were then fabricated as followings: after deposition of $0 \sim 30$ Athick UTA-Si, the $\mathrm{Si}_{1-x} \mathrm{Ge}_{x}(x \approx 0.25)$ alloy layer was deposited during the gradual increase in substrate temperature from 300 to $500^{\circ} \mathrm{C}$ at a rate of $20^{\circ} \mathrm{C} / \mathrm{min}$, then the rest was deposited at $500^{\circ} \mathrm{C}$. The total thickness of SiGe layer is $200 \mathrm{~nm}$. During the increase in the substrate with UTA-Si to $300^{\circ} \mathrm{C}$, the UTA-Si may be slightly crystallized, giving the possibility of epitaxial growth of SiGe layer.

The surface morphology was observed by atomic force microscope (AFM) (digital instruments, nanoscope-III) and the surface roughness was characterized by root- mean-squared (RMS) roughness of the AFM images. The structural and crystal-quality analyses were performed on a Rigaku ATX-E diffractometer (at a wavelength of $1.5406 \AA)$, which is equipped with a four-crystal Ge(220) monochromator and a channel-cut $\mathrm{Ge}(220)$ analyzer. The independent variation of the two diffraction angles $\omega$ (between primary X-ray beam and the sample surface) and $2 \theta$ (between incident and diffracted X-ray beams) provides the possibility of HRRSM, i.e. two-dimensionally resolved measurements of distributions of diffracted X-ray intensity.

\section{RESULTS AND DISCUSSIONS}

Figure 1 shows the reciprocal space coordinates. The Ewald sphere with radius $1 / \lambda$ illustrates the path of incident and diffracted beams. The selected quadrangle around the $(h k l)$ reciprocal lattice points is the regions for two axes scan, which are illustrated on it in $\omega$ and 


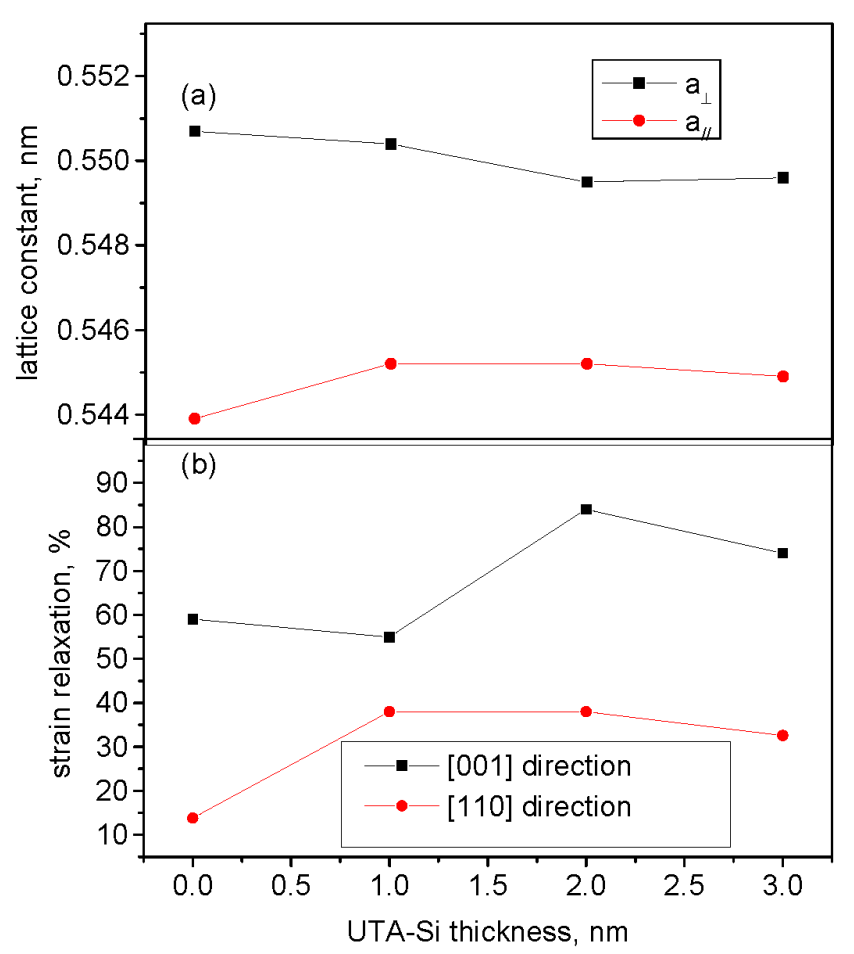

FIG. 3: (a) The out-of-plane lattice constant $a_{\perp}$ and the inplane lattice constant $a_{/ /}$of SiGe calculating from (113) reciprocal lattice point and (b) the degree of the strain-relaxation

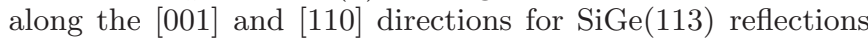
in samples with UTA-Si with a various thickness.

$2 \theta / \omega$ scans. In the figure, the coordinate position $\left(q_{x}, q_{y}\right)$ of $(h k l)$ reciprocal lattice point is characterized by its $\omega$ and $2 \theta / \omega$ values, $q_{x}$ is parallel to the direction of incident $\mathrm{X}$-ray, and $q_{y}$ is perpendicular to the surface. From the geometry of the reciprocal lattice and Ewald construction, we can calculate the reciprocal lattice vector components, $q_{x}$ and $q_{y}$ :

$$
\begin{aligned}
& q_{x}=\frac{2}{\lambda} \sin \theta \cdot \sin (\theta-\omega), \\
& q_{y}=\frac{2}{\lambda} \sin \theta \cdot \cos (\theta-\omega),
\end{aligned}
$$

where $\lambda$ is the X-ray wavelength, $\omega$ the angle between primary X-ray beam and the sample surface, and $2 \theta$ the angle between incident and diffracted X-ray beams.

In the principle of reciprocal lattice, the length of the reciprocal lattice vector $\vec{Q}(h k l)$, from the origin (000) to point $(h k l)$, is just the reciprocal of the interplanar spacing $d(h k l)$ for the planes $(h k l)$, i.e. $d(h k l)=1 /|\vec{Q}(h k l)|$. So, the out-of-plane lattice constant, $a_{\perp}$, and in-plane lattice constant, $a_{/ /}$, can be calculated by $a_{\perp}=3 / q_{y}$ and $a_{/ /}=\sqrt{2} / q_{x}$ in the case of (113) point and $a_{\perp}=4 / q_{y}$ in the case of (004) point.

Figures 2(a) and (b) show the typical experimental reciprocal space maps of the (004) and (113) reflections from the samples, where (a) SiGe/Si, and (b) SiGe/UTA-Si(20 $\AA) /$ Si. The diffraction intensities are plotted with a logarithmic scale as functions of the reciprocal space vector components, $q_{x}$ and $q_{y}$. The inner fan-shaped quadrangle is the two axes scan region. Because the lattice constant of

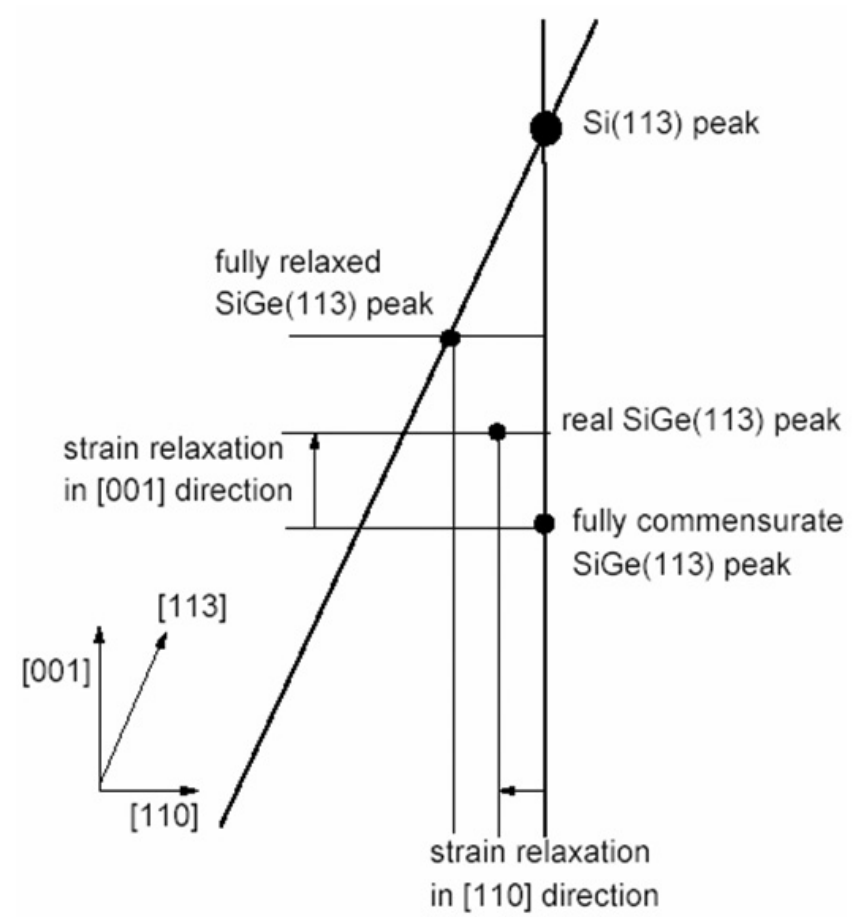

FIG. 4: The schematic diagram to calculate the degree of strain relaxation in [110] and [001] directions from $\operatorname{SiGe(113)}$ diffraction peak obtained from HRRSM.

SiGe is larger than $\mathrm{Si}$, the reciprocal lattice point of SiGe is located lower than Si. We can easily find the maximum diffraction intensity point as the Si center position to be the reference position. The diffraction peaks of SiGe without UTA-Si exhibit very broadening not only in the $\omega$ scan but also in $2 \theta / \omega$ scan, and the diffraction intensity center is difficult to find out. After using UTA-Si, the scattering distributions width both in $\omega$ scan and $2 \theta / \omega$ scan direction reduce, which indicates an improvement of the crystal quality. The SiGe diffraction peaks exhibit broadening in the $\omega$ scan indicated the formation of mosaics structure. The mosiacity in the layer means that there are angular variations in the (004) or (113) directions of the planes of the layer due to relaxation by generation of misfit dislocations. By determining the maximum intensity positions of SiGe (004) reciprocal lattice points, we found the center position of $\mathrm{SiGe}$ reciprocal lattice point was in the $2 \theta / \omega$ scan direction perfectly, which indicate that there is no crystalline tilt between $\mathrm{Si}$ and $\mathrm{SiGe}$. After comparing the diffraction points of SiGe with different thickness of UTA-Si buffer layers, we found that the diffraction peak scattering distributions of SiGe with $1 \mathrm{~nm}$ thick UTA-Si is broader in $2 \theta / \omega$ scan direction and narrower in $\omega$ scan direction than those of samples with $2 \mathrm{~nm}$ or $3 \mathrm{~nm}$ thick UTA-Si, which mean that the strain relaxation of the samples with 2 or $3 \mathrm{~nm}$ thick UTA-Si is higher than that with 1nm thick UTA-Si. The increase of the UTA-Si thickness will decrease the mosaic spread, which coincides with the strain transfer and relaxation of the SiGe alloy [10].

From the typical HRRSMs around asymmetric (113) reflections from SiGe /UTA-Si/Si, we can draw the same conclusions about the crystal quality. But we find that without UTA-Si buffer layer, the center positions of 


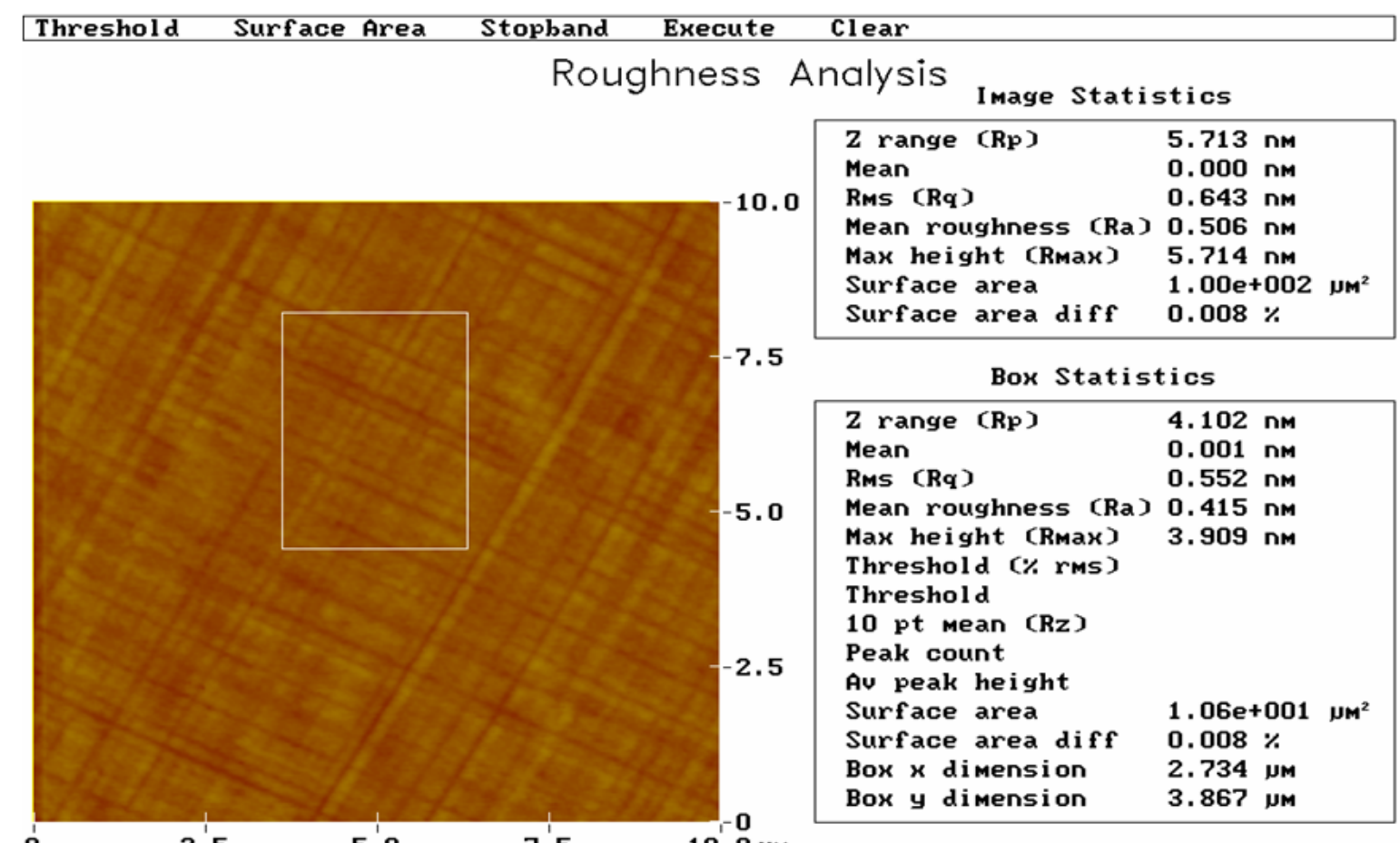

Thresh cal off Area on

FIG. 5: The surface morphology and roughness analysis of SiGe alloy layer grown using UTA-Si as buffer layer.

SiGe and Si reciprocal lattice points are almost perfectly aligned in the $q_{\perp}$ direction showing that there is a little strain relaxation in [110] direction. After using UTA-Si, the position of the (113) SiGe layer peak is shifted toward the $2 \theta / \omega$ axis, indicative of increase of strain relaxation in [110] direction. At the same time, we find that the center position of SiGe layer changed as a function of the thickness of UTA-Si, which indicate the change of the out-ofplane lattice constant and in-plane lattice constant, corresponding to the strain relaxation. We can calculate the in-plane lattice constant and out-of-plane lattice constant using the relationships between the lattice constants and the reciprocal space coordinates. The results are shown in Fig. 3(a). The calculated values of $a_{/ /}$and $a_{\perp}$ can be used to determine the degree of relaxation in-plane layer and out-of-plane layer, respectively.

Here, we introduce a simple method to determine the relative strain relaxation. As shown in Fig. 4, we assume that the degree of strain relaxation with the lattice constant of bulk SiGe is $100 \%$, and that of pseudomorphic SiGe is $0 \%$. The fully relaxed $\mathrm{SiGe}$ peak point is located in $2 \theta / \omega$ scan axis, which can be ascertained combined with bulk SiGe lattice constant $a_{0}=0.5485 \mathrm{~nm}$ for $\mathrm{Si}_{0.75} G e_{0.25}$ [11]; the pseudomorphic SiGe has the same in-plane lattice constant as $\mathrm{Si}$, so its peak point can be decided combined with the $a_{\perp}$ value calculated from the relationship $a_{\perp}=\frac{1+\gamma}{1-\gamma} a_{0}-\frac{2 \gamma}{1-\gamma} a_{/ /}$, in which $\gamma$ is the Poisson ratio [12]. Figure 4 explains our analytical method for obtaining the degree of the strain-relaxation in the SiGe layers estimated from the real peak position of $\mathrm{SiGe}(113)$ reflection in the two-dimensional reciprocal-space map. Since the completely-relaxed and the completely-strained
SiGe(113) peak positions are explicitly known in the figure, the degree of strain relaxation of the (113) plane can be obtained from the measured diffraction peak position correspondingly. Furthermore, the strain in the asymmetric (113) plane can be divided into the [001] and the [110] components. So, the degree of strain relaxation in [001] and [110] directions can be calculated. Figure 3(b) is the result of this analysis showing the degree of the strain relaxation in the $\mathrm{SiGe}$ layers as a function of the UTA-Si thickness. We find that the residual strain in the SiGe layer can be decreased by increasing the thickness of the UTA-Si. The $84 \%$ strain relaxation is achieved for the sample with the $2 \mathrm{~nm}$ thick UTA-Si along the [001] direction while $38 \%$ strain relaxation along the [110] direction. The difference of the strain relaxation between those along [001] and [110] directions is probably caused by the structure of $60^{\circ}$ or $90^{\circ}$ dislocations.

The existence of misfit dislocation can also be approved in AFM images, because the crosshatch pattern characteristic of SiGe surface is corresponding to misfit dislocations [13-17]. Figure 5 shows the AFM image of the sample with $2 \mathrm{~nm}$ thick UTA-Si, where a RMS surface roughness is equal to $0.643 \mathrm{~nm}$ for scanning areas of $10 \times 10$ $\mu \mathrm{m}^{2}$, the sides of the images are parallel to the [001] and [010], respectively. We find that the crosshatch pattern on the grown sample is evident from the atomic force micrograph, which is evidence of the response of the growth surface to the underlying misfit dislocation indicating the relaxation of strain. Though there is crosshatch on the surface, the $0.643 \mathrm{~nm}$ RMS roughness of the surface is comparable to that of Si substrate. 


\section{CONCLUSIONS}

As a sophisticated experimental technique, highresolution X-ray reciprocal space mapping has been used for the studies of strain-relaxation and crystallinity of SiGe films using UTA-Si buffer layer. Measured results have shown a clear improvement in surface roughness and strain relaxation of SiGe thin film. UTA-Si buffer layer can not only save the fabrication cost and time but also meet special utilization. At the same time, the strain relaxation and the RMS roughness comparable to Si substrate make it possible to be used as a virtual substrate to fabricate high quality devices, because such SiGe is the base for the growth of the strained $\mathrm{Si} / \mathrm{SiGe}$ hetero-devices, which are attracting much attention due to the possibility to largely enhanced mobility and improved device performances $[18,19]$.
[1] C. Tatsuyama, T. Asano, T. Nakao, H. Matada, T. Tambo, and H. Ueba, Thin Solid Film 369, 161 (2000).

[2] J. M. Hartmann, B. Gallas, J. Zhang, J. J. Harris and B. A. Joyce, J. Appl. Phys. 86, 845 (1999).

[3] M. M. Rahman, K. Kurumatani, H. Matada, T. Tambo and C. Tatsuyama, Mater. Sci. Engi. B 89, 252 (2002).

[4] M. M. Rahman, H. Matada, T. Tambo and C. Tatsuyama, J. Appl. Phys. 90, 202 (2001).

[5] M. Rahman, K. Kurumatani, H. Matada, T. Tambo and C. Tatsuyama, Jpn. J. Appl. Phys. 41, 2845 (2002).

[6] C. S. Peng, Z. Y. Zhao, H. Chen, J. H. Li, Y. K. Li, L. W. Guo, D. Y. Dai, Q. Huang, Y. H. Zhang, T. T. Sheng and C. H. Tung, Appl. Phys. Lett. 72, 3160 (1998).

[7] C. S. Peng, Y. K. Li, Q. Huang and J. M. Zhou, J. Crystal Growth, 227-228, 740 (2001).

[8] S. W. Lee, H. C. Chen, L. J. Chen, Y. H. Peng, C. H. Kuan and H. H. Cheng, J. Appl. Phys. 92, 6880 (2002).

[9] Y. H. Luo, J. Wan, R. L. Forrest, J. L. Liu, M. S. Goorsky and K. L. Wang, J. Appl. Phys. 89, 8279 (2001).

[10] F. Y. Huang, M. A. Chu, M. O. Tanner, K. L. Wang G. D. UfRen and M. S. Goorsky, Appl. Phys. Lett. 76, 2680 (2000).
[11] J. P. Dismukes, L. Ekstrom, and R. J. Paff, J. Phys. Chem. 68, 3021 (1964)

[12] S. Hong, Y. L. Foo, K. A. Bratland, T. Spila, K. Ohmori, M. R. Sardela, J. E. Greene and E. Yoon, Appl. Phys. Lett., 83, 4321 (2003).

[13] E. A. Fitzgerald, Y. H. Xie, D. Monroe, J. Vac. Sci. Technol. B 10, 1807 (1992).

[14] J. W. P. Hsu, E. A. Fitzgerald, Y. H. Xie, P. J. Silverman, M. J. Cardillo, Appl. Phys. Lett. 61, 1293 (1992).

[15] S. Shiryaev, F. Jensen, J. W. Petersen, Appl. Phys. Lett. 64, 3305 (1994)

[16] M. A. Lutz, R. M. Feenstra, F. K. LeGoues, P. M. Mooney, J. O. Dhu, Appl. Phys. Lett. 66, 724 (1995).

[17] M. Albrecht, S. Christiansen, J. Michler, Appl. Phys. Lett. 67, 1232 (1995).

[18] Y. J. Mii, Y. H. Xie, E. A. Fitzgerald, D. Monroe, F. A. Thiel, B. E. Weir, L. C. Feldman, Appl. Phys. Lett. 59, 1611 (1991).

[19] Kismail, F. K. LeGoues, K. L. Saenger, Marafa, J. O. Chu, P. M. Mooney, B. S. Meyerson, Phys. Rew. Lett. 73, 3447 (1994). 